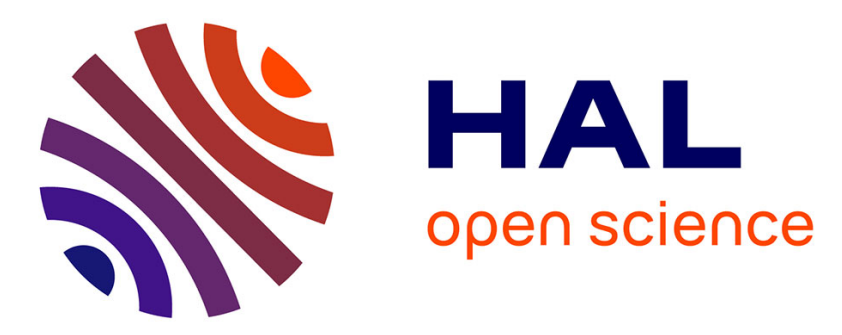

\title{
Monolithic approach of Stokes-Darcy coupling for LCM process modelling
}

Lara Abouorm, Nicolas Moulin, Julien Bruchon, Sylvain Drapier

\section{To cite this version:}

Lara Abouorm, Nicolas Moulin, Julien Bruchon, Sylvain Drapier. Monolithic approach of StokesDarcy coupling for LCM process modelling. CURRENT STATE-OF-THE-ART ON MATERIAL FORMING: NUMERICAL AND EXPERIMENTAL APPROACHES AT DIFFERENT LENGTHSCALES, PTS 1-3, 2013, 554-557, pp.447-455. 10.4028/www.scientific.net/KEM.554-557.447 . emse00959587

\section{HAL Id: emse-00959587 \\ https://hal-emse.ccsd.cnrs.fr/emse-00959587}

Submitted on 24 Aug 2021

HAL is a multi-disciplinary open access archive for the deposit and dissemination of scientific research documents, whether they are published or not. The documents may come from teaching and research institutions in France or abroad, or from public or private research centers.
L'archive ouverte pluridisciplinaire HAL, est destinée au dépôt et à la diffusion de documents scientifiques de niveau recherche, publiés ou non, émanant des établissements d'enseignement et de recherche français ou étrangers, des laboratoires publics ou privés. 


\title{
Monolithic approach of Stokes-Darcy coupling for LCM process modelling
}

\author{
Lara Abouorm $^{1, \mathrm{a}}$, Nicolas Moulin ${ }^{1, \mathrm{~b}}$, Julien Bruchon ${ }^{1, \mathrm{c}}$ and Sylvain Drapier ${ }^{1, \mathrm{~d}}$ \\ LCG UMR CNRS 5146, Centre SMS, Ecole Nationale Supérieure des Mines de Saint-Etienne, \\ 158 cours Fauriel, 42023 Saint-Etienne cedex 2, France

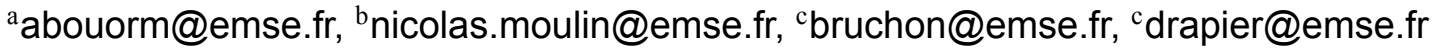

Keywords: Darcy, Stokes, composites, level set, stabilization, multi-scale method.

\begin{abstract}
The present contribution is devoted to developing robust finite element solutions for coupling flows in both purely fluid region, ruled by Stokes equations, and fibrous preform region governed by a Darcy's law. Particularly the cases of low permeability of preform, down to $10^{-15} \mathrm{~m}^{2}$, are of interest to model LCM processes. Flows are solved using mixed finite elements stabilized with a sub-grid scale stabilization technique (ASGS). A special attention is paid to the interface conditions, namely normal stress and velocity continuity and tangential velocity constraint similar to a Beaver-JosephSaffman's condition. The originality of the model consists in using one single mesh to represents the Stokes and the Darcy sub-domains (monolithic approach). A level set context is used to represent Stokes-Darcy interface and to capture the moving flow front. It is shown that provided a very special attention is paid to the coupling conditions, very precise results can be obtained. This monolithic approach is now perfectly robust due to the introduction of the ASGS sub-grid scale stabilization and leads to perform $3 \mathrm{D}$ complex shapes for manufacturing process by resin infusion.
\end{abstract}

\section{Introduction}

Manufacturing processes by resin infusion are competitive routes to elaborate composite structures with organic matrix, especially for large pieces in aeronautics. They consist in infusing a liquid resin through the thickness of the reinforcements rather than in their plane. Under the effect of a mechanical pressure applied on the top of the mold, the resin flows and infuses into the preforms. However, although these processes are efficient, they still remain hard to control. Indeed the physical and mechanical properties of the final part (the final thickness and the fiber volume) are hardly predictable. To control this process, we developed a model based on the coupling between the resin flow within the porous domain (Darcy), and the purely fluid domain (Stokes). The Stokes-Darcy coupled problem has been studied by many researchers in many field of engineering. Both a decoupled approach as proposed by [1] and a monolithic approach as proposed by [2] are investigated in severe regimes. The decoupled strategy consists of using two different element spaces to solve the Stokes and Darcy equations, whereas the unified strategy consists in using the same finite element space. In literature, flows are solved using mixed finite elements stabilized with P1+/P1 elements in Stokes and Darcy's domain [1]. Another approach consists in using P1+/P1 elements in Stokes and HVM (Hughes Variational Multiscale) Method for stabilization in Darcy [2]. Due to consistency errors and spurious oscillations that appear in this previous approach, we use a robust approach which yields improvements compared to the latest one. The robustness of the approach which is assessed in this paper, is ensured by using ASGS method (Algebraic Subgrid Scale) to stabilize velocity and pressure approximated by linear and continuous elements in Stokes and Darcy domains. Signed functions are used to represent the Stokes-Darcy interface and to capture the moving flow front.

The paper is organized as follows. The first section presents the mathematical modelling for the Stokes-Darcy coupled problem. The next section introduces both the velocity-pressure mixed formulation for the Stokes-Darcy problem and the variational multiscale method used for stabilization. The last section shows numerical validation and results in severe regimes (low permeability, down to $10^{-15} \mathrm{~m}^{2}$, complex 2D and 3D geometries with curved interfaces) to illustrate the capability of modelling manufacturing processes by resin infusion. 


\section{Mathematical model}

Let us define $\Omega \in R^{m}$ ( $m=2$ or $m=3$ ) a bounded domain formed by two non overlapping subdomains $\Omega_{s}$ and $\Omega_{d}$ separated by a surface $\Gamma=\partial \Omega_{s} \cap \partial \Omega_{d}$. Index $s$ is used to denote everything that concerns the purely fluid part (Stokes domain), and index $d$ for modelling porous medium (Darcy domain). In this section, we present Stokes and Darcy equations, then we present the interface conditions on $\Gamma$ and the coupling of the two domains. The fluid is considered as Newtonian and incompressible. The Stokes equations are then expressed as: find the velocity $\mathbf{v}_{\mathbf{s}}$ and the pressure $p_{s}$ fields defined by :

$$
\begin{aligned}
-\operatorname{div}\left(2 \mu \dot{\boldsymbol{\varepsilon}}\left(\mathbf{v}_{\mathbf{s}}\right)\right)+\nabla p_{s} & =\mathbf{f}_{\mathbf{s}} \text { in } \Omega_{s} \\
-\operatorname{div} \mathbf{v}_{\mathbf{s}} & =h_{s} \text { in } \Omega_{s} \\
\mathbf{v}_{\mathbf{s}} & =\mathbf{v}^{1} \text { on } \Gamma_{s, D} \\
\boldsymbol{\sigma}_{\boldsymbol{n}} & =p_{\text {ext, }, s} \cdot \mathbf{n}_{\mathbf{s}} \text { on } \Gamma_{s, N}
\end{aligned}
$$

where $\mathbf{f}_{\mathbf{s}}$ is the volume force, $\mathbf{n}_{\mathbf{s}}$ is the unit vector normal to the boundary of $\Omega_{s}$ and $\sigma_{n}$ is the stress vector to be prescribed on $\Gamma_{s, N}$. If the fluid is incompressible then $h_{s}=0$.

The Darcy equations are expressed as: find the velocity $\mathbf{v}_{\mathbf{d}}$ and the pressure $p_{d}$ defined on $\Omega_{d}$, solution of :

$$
\begin{aligned}
\frac{\mu}{\mathrm{k}} \mathbf{v}_{\mathbf{d}}+\nabla p_{d} & =\mathbf{f}_{\mathbf{d}} \text { in } \Omega_{d} \\
-\operatorname{div} \mathbf{v}_{\mathbf{d}} & =h_{d} \text { in } \Omega_{d} \\
\mathbf{v}_{\mathbf{d}} \cdot \mathbf{n}_{\mathbf{d}} & =g \text { on } \Gamma_{d, D} \\
p_{d} & =p_{e x t, d} \quad \text { on } \Gamma_{d, N}
\end{aligned}
$$

where $\mathrm{k}$ is the permeability tensor reduced to a scalar in the isotropic case considered here, $p_{\text {ext, }}$ is a pressure to be prescribed on $\Gamma_{d, N}, \mathbf{n}_{\mathbf{d}}$ is the outward unit vector normal to the boundary of $\Omega_{d}$ and $\mathbf{f}_{\mathbf{d}}$ is the volume force. If the fluid is incompressible then $h_{d}=0$. In Stokes-Darcy coupled problem, boundary conditions must be considered on the interface $\Gamma$ of normal $n=n_{s}$. These conditions are:

\section{Continuity of normal velocity}

The mass conservation through interface $\Gamma$ is expressed by the continuity of the velocity field $\mathbf{v}$ across $\Gamma$ :

$$
\mathbf{v}_{\mathbf{s}} \cdot \mathbf{n}_{\mathbf{s}}+\mathbf{v}_{\mathbf{d}} \cdot \mathbf{n}_{\mathbf{d}}=0 \text { on } \Gamma
$$

Continuity of the fluid normal stress

$$
\mathbf{n} \cdot \boldsymbol{\sigma}_{s} \cdot \mathbf{n}=\mathbf{n} \cdot \boldsymbol{\sigma}_{d} \cdot \mathbf{n} \text { on } \Gamma
$$

\section{Beaver-Joseph-Saffman condition}

The Beaver Joseph Saffman condition allows the tangential velocity to be specified on the interface

$\Gamma$. The interface condition can be written as:

$$
2 \mathbf{n} \cdot \dot{\varepsilon}\left(\mathbf{v}_{\mathbf{s}}\right) \cdot \boldsymbol{\tau}_{j}=\frac{\alpha}{\sqrt{\mathrm{k}}}\left(\mathbf{v}_{\mathbf{s}} \cdot \boldsymbol{\tau}_{j}\right)
$$

where $\alpha$ is a dimensionless parameter, so-called slip coefficient and $\tau_{j}$ are the tangential vectors on the interface. 


\section{Weak formulation}

In order to solve the Stokes-Darcy coupled problem by a finite element method, the weak formulation has to be established. We present the weak formulation of Stokes and Darcy's systems separately. The weak formulation of the coupled problem is obtained by summing up the Stokes and Darcy's weak formulations and by taking into consideration interface conditions described in the Mathematical model section. For a sake of simplicity, we choose to write the $L^{2}$ inner product in $\Omega_{d / s}$ as $<\cdot, \cdot>$. The following functional spaces are used as velocity, pressure and test function spaces:

$$
\begin{aligned}
L^{2}\left(\Omega_{i}\right) & =\left\{u: \Omega_{i} \rightarrow R \mid \int_{\Omega_{i}} u^{2} d \Omega<\infty\right\} \\
H^{1}\left(\Omega_{i}\right)^{m} & =\left\{u \in L^{2}\left(\Omega_{i}\right)^{m} \mid \nabla u \in L^{2}\left(\Omega_{i}\right)^{m \times m}\right\} \\
H_{\Gamma_{i, D}}^{1}\left(\Omega_{i}\right)^{m} & =\left\{u \in H^{1}\left(\Omega_{i}\right)^{m} \mid u=u^{1} \text { on } \Gamma_{i, D}\right\} \\
H_{\Gamma_{i, D}}^{1, t}\left(\Omega_{i}\right)^{m} & =\left\{u \in H^{1}\left(\Omega_{i}\right)^{m} \mid u=0 \text { on } \Gamma_{i, D}\right\} \\
H\left(d i v, \Omega_{i}\right) & =\left\{u \in L^{2}\left(\Omega_{i}\right)^{m} \mid \operatorname{div} u \in L^{2}\left(\Omega_{i}\right)\right\} \\
H_{\Gamma_{i, D}}\left(\operatorname{div}, \Omega_{i}\right) & =\left\{u \in H\left(\operatorname{div}, \Omega_{i}\right) \mid u . \mathbf{n}=u^{2} \text { on } \Gamma_{i, D}\right\} \\
H_{\Gamma_{i, D}}^{t}\left(\operatorname{div}, \Omega_{i}\right) & =\left\{u \in H\left(\operatorname{div}, \Omega_{i}\right) \mid u \cdot \mathbf{n}=0 \text { on } \Gamma_{i, D}\right\}
\end{aligned}
$$

with $i=s$ or $i=d$ and $m$ is the dimension equal to 2 or 3 .

The variational formulation of Stokes problem consists in finding a velocity-pressure

$$
\begin{aligned}
& \text { pair }\left[\mathbf{v}_{\mathbf{s}}, p_{s}\right] \in H_{\Gamma_{s, D}}^{1}\left(\Omega_{i}\right)^{m} \times L^{2}\left(\Omega_{s}\right), \text { such that : } \\
& B_{s}\left(\left[\mathbf{v}_{\mathbf{s}}, p_{s}\right],\left[\mathbf{w}_{\mathbf{s}}, q_{s}\right]\right)=L_{s}\left(\left[\mathbf{w}_{\mathbf{s}}, q_{s}\right]\right), \quad \forall \mathbf{w}_{\mathbf{s}} \in H_{\Gamma_{s, D}}^{1, t}\left(\Omega_{s}\right)^{m}, \forall q_{s} \in L^{2}\left(\Omega_{s}\right) .
\end{aligned}
$$

where the bilinear form $B_{s}$ and the linear form $L_{s}$ are defined in Stokes by:

$$
\begin{aligned}
B_{s}\left(\left[\mathbf{v}_{\mathbf{s}}, p_{s}\right],\left[\mathbf{w}_{\mathbf{s}}, q_{s}\right]\right)= & <2 \mu \dot{\varepsilon}\left(\mathbf{v}_{\mathbf{s}}\right): \dot{\varepsilon}\left(\mathbf{w}_{\mathbf{s}}\right)>_{\Omega_{s}}-<p_{s}, \operatorname{div} \mathbf{w}_{\mathbf{s}}>_{\Omega_{s}}-<\operatorname{div} \mathbf{v}_{\mathbf{s}}, q_{s}>_{\Omega_{s}} \\
& +<p_{d}, \mathbf{w}_{\mathbf{s}} \cdot \mathbf{n}_{\mathbf{s}}>_{\Gamma}+<\frac{\alpha \eta}{\sqrt{\mathrm{k}}}\left(\mathbf{v}_{\mathbf{s}} \cdot \boldsymbol{\tau}\right),\left(\mathbf{w}_{\mathbf{s}} \cdot \boldsymbol{\tau}\right)>_{\Gamma} \\
L_{s}\left(\left[\mathbf{w}_{\mathbf{s}}, q_{s}\right]\right)= & <\mathbf{f}_{\mathbf{s}}, \mathbf{w}_{\mathbf{s}}>_{\Omega_{s}}+<h_{s}, q_{s}>_{\Omega_{s}} \\
& +<p_{\text {ext }, s} \cdot \mathbf{n}_{\mathbf{s}}, \mathbf{w}_{\mathbf{s}}>_{\Gamma_{s, N}}
\end{aligned}
$$

The variational formulation of Darcy's problem consists in finding a velocity-pressure

$$
\begin{aligned}
& \text { pair }\left[\mathbf{v}_{\mathbf{d}}, p_{d}\right] \in H_{\Gamma_{d, D}}\left(\operatorname{div}, \Omega_{d}\right) \times L^{2}\left(\Omega_{d}\right) \text { such that : } \\
& B_{d}\left(\left[\mathbf{v}_{\mathbf{d}}, p_{d}\right],\left[\mathbf{w}_{\mathbf{d}}, q_{d}\right]\right)=L_{d}\left(\left[\mathbf{w}_{\mathbf{d}}, q_{d}\right]\right), \quad \forall \mathbf{w}_{\mathbf{d}} \in H_{\Gamma_{d, D}}^{t}\left(\operatorname{div}, \Omega_{d}\right), \forall q_{d} \in L^{2}\left(\Omega_{d}\right) .
\end{aligned}
$$

The bilinear form $B_{d}$ and the linear form $L_{d}$ are defined in Darcy by :

$$
\begin{aligned}
B_{d}\left(\left[\mathbf{v}_{\mathbf{d}}, p_{d}\right]\left[\mathbf{w}_{\mathbf{d}}, q_{d}\right]\right)= & \frac{\mu}{\mathrm{k}}<\mathbf{v}_{\mathbf{d}}, \mathbf{w}_{\mathbf{d}}>_{\Omega_{d}}-<p_{d}, \operatorname{div} \mathbf{w}_{\mathbf{d}}>_{\Omega_{d}}-<q_{d}, \operatorname{div} \mathbf{v}_{\mathbf{d}}>_{\Omega_{d}} \\
& -<p_{d}, \mathbf{w}_{\mathbf{s}} \cdot \mathbf{n}_{\mathbf{s}}>_{\Gamma} \\
L_{d}\left(\left[\mathbf{w}_{\mathbf{d}}, q_{d}\right]\right)= & <\mathbf{f}_{\mathbf{d}}, \mathbf{w}_{\mathbf{d}}>_{\Omega_{d}}+<h_{d}, q_{d}>_{\Omega_{d}}+<p_{e x t, d} \cdot \mathbf{n}, \mathbf{w}_{\mathbf{d}}>_{\Gamma_{d, N}}
\end{aligned}
$$


The mixed formulation of the Stokes-Darcy problem is established by considering a velocity $\mathbf{v}$ on $\Omega$ and a pressure field $p$ on $\Omega$ such as $\mathbf{v}_{/ \Omega_{i}}=\mathbf{v}_{i}$ and $p_{/ \Omega_{i}}=p_{i}$ with $i=s$ or $i=d$. The mixed weak formulation of Stokes-Darcy is obtained by summing up Eq. 8 and Eq. 11 and taking into consideration the conditions imposed on the Stokes-Darcy interface described in Mathematical model section. Hence, the variational formulation of the Stokes-Darcy coupled problem consist in finding $[\mathbf{v}, p] \in V \times Q$ such that :

$$
B_{c}[(\mathbf{v}, p),(\mathbf{w}, q)]=L_{c}\left([\mathbf{w}, q], \quad \forall[\mathbf{w}, q] \text { defined in } V_{0} \times Q\right.
$$

with:

$$
\begin{aligned}
V & =H_{\Gamma_{s, D}}^{1} \times H_{\Gamma_{d, D}}\left(\operatorname{div}, \Omega_{d}\right) \\
V_{0} & =H_{\Gamma_{s, D}}^{1, t} \times H_{\Gamma_{d, D}}^{t}\left(\operatorname{div}, \Omega_{d}\right) \\
Q & =L^{2}\left(\Omega_{s}\right) \times L^{2}\left(\Omega_{d}\right)
\end{aligned}
$$

The bilinear form $B_{c}$ and the linear form $L_{c}$ are defined by:

$$
\begin{aligned}
B_{c}([\mathbf{v}, q],[\mathbf{w}, q])= & <2 \mu \dot{\varepsilon}(\mathbf{v}): \dot{\varepsilon}(\mathbf{w}) H_{s}>_{\Omega}+<\frac{\mu}{\mathbf{k}} \mathbf{v}, \mathbf{w} H_{d}>_{\Omega}-<p, \operatorname{div} \mathbf{w}>_{\Omega} \\
& -<q, \operatorname{div} \mathbf{v}>_{\Omega}+<\frac{\alpha \mu}{\sqrt{\mathrm{k}}} \mathbf{v}, \mathbf{w}>_{\Gamma} \\
L_{c}([\mathbf{v}, p],[\mathbf{w}, q])= & <\mathbf{f}, \mathbf{w}>_{\Omega}+<h, q>_{\Omega}+<p_{\text {ext, } s} . \mathbf{n}, \mathbf{w}>_{\Gamma_{s, N}}+<p_{\text {ext,d }} . \mathbf{n}, \mathbf{w}>_{\Gamma_{d, N}}
\end{aligned}
$$

(f, $h$ ) are defined by $\left(\mathbf{f}_{\mathbf{i}}, h_{i}\right)$ in $\Omega_{i} . H_{i}$ is the Heaviside function, equal to 1 in domain $i$ and vanishing elsewhere.

\section{Finite element method for Stokes-Darcy coupled problem}

The whole domain $\Omega \subset \mathrm{R}^{\mathrm{m}}$ is discretized with one single unstructured mesh. This mesh is made up of triangles if $m=2$ and of tetrahedrons if $m=3$. Let $V_{h}$ and $Q_{h}$ be the finite element spaces of the piecewise linear and continuous functions, which contains the solutions $\mathbf{v}_{\mathbf{h}, \mathbf{i}}$ and $p_{h, i}$. The Galerkin approximation of both the Stokes and the Darcy problems requires the use of velocity-pressure interpolation that satisfy the adequate inf sup condition. Different interpolations pairs are known to satisfy this condition for each problem independently, but the key issue is to find interpolations that satisfy both at the same time. In this paper, we choose the use of stabilized finite element methods. The philosophy of the stabilized methods is to strengthen classical variational formulations so that discrete approximation which would otherwise be unstable becomes stable and convergent. One of the stabilized finite element method approximation for Stokes-Darcy problem is VMS (Variational Multiscale Method) [3, 4]. The basic idea of this method is to approximate the effect of the component of the continuous solution which cannot be captured by the finite element solution. It consists in splitting the continuous solution for velocity and pressure into two components, one coarse corresponding to the finite element scale $\left[\mathbf{v}_{\mathbf{h}, \mathbf{i}}, p_{h, i}\right]$, and a finer component corresponding to lower scale $\left[\mathbf{v}_{\mathbf{i}}^{\prime}, p_{i}^{\prime}\right]$ for resolutions. The velocity is approximated as $\mathbf{v}_{\mathbf{i}}=\mathbf{v}_{\mathbf{h}, \mathbf{i}}+\mathbf{v}_{\mathbf{i}}^{\prime}$ and the pressure field is approximated as $p_{i}=p_{h, i}+p_{i}^{\prime}$. We consider a subgrid space $V \times Q=\left(V_{h} \times Q_{h}\right) \bigoplus\left(V^{\prime} \times Q^{\prime}\right)$. Invoking this decomposition in the continuous problem for both the solution and test functions, the finite element problem becomes [4]:

$$
B_{s}\left(\left[\mathbf{v}_{\mathbf{h}, \mathbf{i}}, p_{h, i}\right],\left[\mathbf{w}_{\mathbf{h}, \mathbf{i}}, q_{h, i}\right]\right)+B_{s}\left(\left[\mathbf{v}_{\mathbf{i}}^{\prime}, p_{i}^{\prime}\right],\left[\mathbf{w}_{\mathbf{h}, \mathbf{i}}, q_{h, i}\right]\right)=L_{s}\left(\left[\mathbf{w}_{\mathbf{h}, \mathbf{i}}, q_{h, i}\right]\right)
$$

The fine scale problem writes:

$$
\left.B_{s}\left(\left[\mathbf{v}_{\mathbf{h}, \mathbf{i}}, p_{h, i}\right],\left[\mathbf{w}_{\mathbf{i}}^{\prime}, q_{i}^{\prime}\right]\right)+B_{s}\left(\left[\mathbf{v}_{\mathbf{i}}^{\prime}, p_{i}^{\prime}\right],\left[\mathbf{w}_{\mathbf{i}}^{\prime}, q_{i}^{\prime}\right]\right)=L_{s}\left(\mathbf{w}_{\mathbf{i}}^{\prime}, q_{i}^{\prime}\right]\right)
$$


for all $\left[\mathbf{w}_{\mathbf{h}, \mathbf{i}}, q_{h, i}\right] \in V_{h} \times Q_{h}$ and $\left[\mathbf{w}_{\mathbf{i}}^{\prime}, q_{i}^{\prime}\right] \in V^{\prime} \times Q^{\prime}$. After approximating (Eq. 17) with an algebraic formulation, by introducing the operator of projection $P^{\prime}$ onto $V^{\prime}$, the approximated fields $\left[\mathbf{v}^{\prime}, p^{\prime}\right]$ are taken into account in the finite element problem (Eq. 16), we get the stabilized forms of the bilinear and linear forms in Stokes, Darcy, and Stokes-Darcy coupled problem. The stabilized problem in Stokes can be written as follow: find $\left[\mathbf{v}_{\mathbf{h}, \mathbf{s}}, p_{h, s}\right] \in V_{h} \times Q_{h}$ such as

$$
B_{s, \text { stable }}\left(\left[\mathbf{v}_{\mathbf{h}, \mathbf{s}}, p_{h, s}\right],\left[\mathbf{w}_{\mathbf{h}, \mathbf{s}}, q_{h, s}\right]\right)=L_{s, \text { stable }}\left(\left[\mathbf{w}_{\mathbf{h}, \mathbf{s}}, q_{h, s}\right]\right)
$$

The bilinear stabilized form $B_{s, \text { stable }}$ and the linear stabilized form $L_{s, s t a b l e}$ are defined by:

$$
\begin{aligned}
B_{s, \text { stable }}\left(\left[\mathbf{v}_{\mathbf{h}, \mathbf{s}}, p_{h, s}\right],\left[\mathbf{w}_{\mathbf{h}, \mathbf{s}}, q_{h, s}\right]\right)= & B_{s}\left(\left[\mathbf{v}_{\mathbf{h}, \mathbf{s}}, p_{h, s}\right],\left[\mathbf{w}_{\mathbf{h}, \mathbf{s}}, q_{h, s}\right]\right) \\
& +\tau_{q} \sum_{K}<\nabla \cdot \mathbf{v}_{\mathbf{h}, \mathbf{s}}, \nabla \cdot \mathbf{w}_{\mathbf{h}, \mathbf{s}}> \\
& +\tau_{v} \sum_{K}<\nabla p_{h, s},-\nabla q_{h, s}> \\
L_{s, \text { stable }}\left(\left[\mathbf{w}_{\mathbf{h}, \mathbf{s}}, q_{h, s}\right]\right)= & L_{s}\left(\left[\mathbf{w}_{\mathbf{h}, \mathbf{s}}, q_{h, s}\right]\right) \\
& -\tau_{q} \sum_{K}<h_{s}, \nabla \cdot \mathbf{w}_{\mathbf{h}, \mathbf{s}}> \\
& +\tau_{v} \sum_{K}<f_{s},-\nabla q_{h, s}>
\end{aligned}
$$

The stabilization parameters $\tau_{v}$ and $\tau_{q}$ are chosen by Fourier transform analysis [4]. $\tau_{v}$ and $\tau_{q}$ are chosen in Stokes by:

$$
\begin{aligned}
\tau_{q} & =c_{1} \mu \\
\tau_{v} & =c_{1} \mu h_{k}^{2}
\end{aligned}
$$

$c_{1}$ is an algorithmic constant and $h_{k}$ is the size of mesh.

By using ASGS method, the stabilized problem in Darcy can be written as follow : find $\left[\mathbf{v}_{\mathbf{h}, \mathbf{d}}, p_{h, d}\right] \in$ $V_{h} \times Q_{h}$ such as

$$
B_{d, \text { stable }}\left(\left[\mathbf{v}_{\mathbf{h}, \mathbf{d}}, p_{h, d}\right]\left[\mathbf{w}_{\mathbf{h}, \mathbf{d}}, q_{h, d}\right]\right)=L_{d, s t a b l e}\left(\left[\mathbf{w}_{\mathbf{h}, \mathbf{d}}, q_{h, d}\right]\right)
$$

with:

$$
\begin{aligned}
B_{d, \text { stable }}\left(\left[\mathbf{v}_{\mathbf{h}, \mathbf{d}}, p_{h, d}\right],\left[\mathbf{w}_{\mathbf{h}, \mathbf{d}}, q_{h, d}\right]\right)= & B_{d}\left(\left[\mathbf{v}_{\mathbf{h}, \mathbf{d}}, p_{h, d}\right],\left[\mathbf{w}_{\mathbf{h}, \mathbf{d}}, q_{h, d}\right]\right) \\
& +\tau_{p} \sum_{K}<\nabla \cdot \mathbf{v}_{\mathbf{h}, \mathbf{d}}, \nabla \cdot \mathbf{w}_{\mathbf{h}, \mathbf{d}}> \\
& +\tau_{u} \sum_{K}<\frac{\mu}{\mathrm{k}} \mathbf{v}_{\mathbf{h}, \mathbf{d}}+\nabla p_{h, d},-\frac{\mu}{\mathrm{k}} \mathbf{w}_{\mathbf{h}, \mathbf{d}}-\nabla q_{h, d}> \\
L_{d, \text { stable }}\left(\left[\mathbf{w}_{\mathbf{h}, \mathbf{d}}, q_{h, d}\right]\right)= & L_{d}\left(\left[\mathbf{w}_{\mathbf{h}, \mathbf{d}}, q_{h, d}\right]\right) \\
& -\tau_{p} \sum_{K}<h_{d}, \nabla \cdot \mathbf{w}_{\mathbf{h}, \mathbf{d}}> \\
& +\tau_{u} \sum_{K}<\mathbf{f}_{\mathbf{d}},-\frac{\mu}{\mathrm{k}} \mathbf{w}_{\mathbf{h}, \mathbf{d}}-\nabla q_{h, d}>
\end{aligned}
$$

where $B_{d}\left(\left[\mathbf{v}_{\mathbf{h}, \mathbf{d}}, p_{h, d}\right],\left[\mathbf{w}_{\mathbf{h}, \mathbf{d}}, q_{h, d}\right]\right)$ and $L_{d}\left(\left[\mathbf{w}_{\mathbf{h}, \mathbf{d}}, q_{h, d}\right]\right)$ are defined in (11). $\tau_{p}, \tau_{u}$ are the stabilization parameters, that we compute as :

$$
\begin{aligned}
\tau_{p} & =c_{p} \frac{\mu}{\mathrm{k}} l_{p}^{2} \\
\tau_{u} & =\left(c_{u} \frac{\mu}{\mathrm{k}} l_{u}^{2}\right)^{-1} h_{k}^{2}
\end{aligned}
$$


with $c_{p}$ and $c_{u}$ algorithmic constants. $l_{u}$ and $l_{p}$ are length scales which can be either taken as $\left(L_{0} h_{k}\right)^{1 / 2}$, $L_{0}$ is a characteristic length of the domain and $h_{k}$ is the element size.

For Stokes and Darcy flow coupled through the interfaces, the stabilized problem with ASGS can be written as follow: find $\left[\mathbf{v}_{\mathbf{h}}, p_{h}\right] \in V_{h} \times Q_{h}$ such as

$$
B_{c, \text { stable }}\left(\left[\mathbf{v}_{\mathbf{h}}, p_{h}\right]\left[\mathbf{w}_{\mathbf{h}}, q_{h}\right]\right)=L_{c, \text { stable }}\left(\left[\mathbf{w}_{\mathbf{h}}, q_{h}\right]\right)
$$

with:

$$
\begin{aligned}
B_{c, s t a b l e}\left(\left[\mathbf{v}_{\mathbf{h}}, p_{h}\right],\left[\mathbf{w}_{\mathbf{h}}, q_{h}\right]\right)= & 2 \mu<H_{s} \dot{\varepsilon}\left(\mathbf{v}_{\mathbf{h}}\right): \dot{\varepsilon}\left(\mathbf{w}_{\mathbf{h}}\right)>_{\Omega}+\frac{\mu}{\mathrm{k}}<H_{d} \mathbf{w}_{\mathbf{h}}, \mathbf{v}_{\mathbf{h}}>_{\Omega} \\
& -<\nabla \cdot \mathbf{w}_{\mathbf{h}}, p_{h}>-<\nabla \cdot \mathbf{v}_{\mathbf{h}}, q_{h}>_{\Omega}+\tau_{p, c}<\nabla \cdot \mathbf{v}_{\mathbf{h}}, \nabla \cdot \mathbf{w}_{\mathbf{h}}>_{\Omega} \\
& +<\alpha \frac{\mu}{\sqrt{\mathrm{k}}}\left(\mathbf{v}_{\mathbf{h}} \cdot \tau\right),\left(\mathbf{w}_{\mathbf{h}} \cdot \tau\right)>_{\Gamma} \\
& +\tau_{u, c}<\frac{\mu}{\mathrm{k}} \mathbf{v}_{\mathbf{h}}+\nabla p_{h},-\frac{\mu}{\mathrm{k}} \mathbf{w}_{\mathbf{h}}-\nabla q_{h}>_{\Omega} \\
L_{c, \text { stable }}\left(\left[\mathbf{w}_{\mathbf{h}}, q_{h}\right]\right)= & <f_{c}, \mathbf{w}_{\mathbf{h}}>_{\Omega}+<h_{c}, q_{h}>_{\Omega}+<p_{\text {ext,s }} \cdot \mathbf{n}, \mathbf{w}_{\mathbf{h}}>_{\Gamma_{s, N}} \\
& +<p_{\text {ext }, d} \cdot \mathbf{n}, \mathbf{w}_{\mathbf{h}}>_{\Gamma_{s, D}}-\tau_{p, c}<h_{c}, \nabla \cdot \mathbf{w}_{\mathbf{h}}>_{\Omega} \\
& +\tau_{u, c}<\mathbf{f}_{\mathbf{c}},-\frac{\mu}{\mathrm{k}} \mathbf{w}_{\mathbf{h}}-\nabla q_{h}>_{\Omega}
\end{aligned}
$$

$\tau_{p, c}, \tau_{u, c}$ are the stabilization parameters, that we compute as:

$$
\begin{aligned}
\tau_{p, c} & =c_{p} \frac{\mu}{\mathrm{k}} l_{p}^{2}+c_{1} \mu \\
\tau_{u, c} & =\left(c_{1} \mu+c_{u} \frac{\mu}{\mathrm{k}} l_{u}\right)^{-1} h_{k}^{2}
\end{aligned}
$$

The surface integral $<\alpha \frac{\mu}{\mathrm{k}}\left(\mathbf{v}_{\mathbf{h}} \cdot \tau\right),\left(\mathbf{w}_{\mathbf{h}} \cdot \tau\right)>_{\Gamma}$ is computed on the interface $\Gamma$ described by the zero isosurface of the signed distance function.

Interface description. The interface $\Gamma$ separating the purely fluid domain and the porous domain is not described by a set of element boundary. This interface passes throughout the mesh elements, consequently a function $\phi$ has to be introduced to depict this interface. $\phi$ is chosen as the signed function to $\Gamma$. Consequently, $\Gamma$ is the zero isosurface of $\phi: \Gamma=\{\phi=0\}$. The level set function $\phi$ which separates Stokes and Darcy's domains allows the computation of the Heaviside functions $H_{s}$ et $H_{d}$ introduced in Eq. 15.

\section{Numerical validation and results}

In this section, the accuracy, the robustness and convergence of the proposed method is investigated. Theoretical orders of convergence are successfully obtained with a super-convergence in some cases due to the optimal choice of the stabilization parameters. These results are not presented in this paper.

In the case of a flow perpendicular (Fig. 1a) to the Stokes/Darcy interface we compare our results with another monolithic approach using MINI-element (P1+/P1) in Stokes and HVM (Hughes Variational Multi-scale) for the stabilization in Darcy [2]. We compare the normal velocity $\mathbf{v}_{\mathbf{y}}$ obtained with ASGS and P1+/P1-HVM methods. In this case, normal velocity should be equal to $10^{-9} \mathrm{~m} / \mathrm{s}$ for a permeability $k=10^{-14} \mathrm{~m}^{2}$. Fig. $1 \mathrm{~b}$ shows the ASGS method assume the continuity of normal velocity and a quasi absence of oscillations while spurious oscillations around the interface and non continuity of normal velocity are obviously seen with P1+/P1-HVM method.

The robustness of ASGS method is demonstrated on more realistic geometries of interest, especially in the context of the manufacturing processes of composite materials. $2 \mathrm{D}$ and $3 \mathrm{D}$ simulations were conducted with a very small height of the Stokes domain with respect to the Darcy's domain height and a very small permeability $\left(\mathrm{k}<10^{-14} \mathrm{~m}^{2}\right)$. 
The figures $2 \mathrm{a}, 2 \mathrm{~b}$ and $2 \mathrm{c}$ show the isovalues of velocity and pressure obtained for the $2 \mathrm{D}$ and $3 \mathrm{D}$ realistic simulations obtained with ASGS method. Despite this curved interface, velocity and pressure fields computed with the monolithic approach do not exhibit any spurious oscillations.

Finally, the mechanical problem with ASGS method is coupled with the level set problem used to capture the resin flow front to simulate the transient fluid flow in Stokes-Darcy domains. A 3D simulation corresponding to a more complex part, essentially to illustrate the $3 \mathrm{D}$ resin flow was conducted. The results of this simulation are shown in Fig. 3.

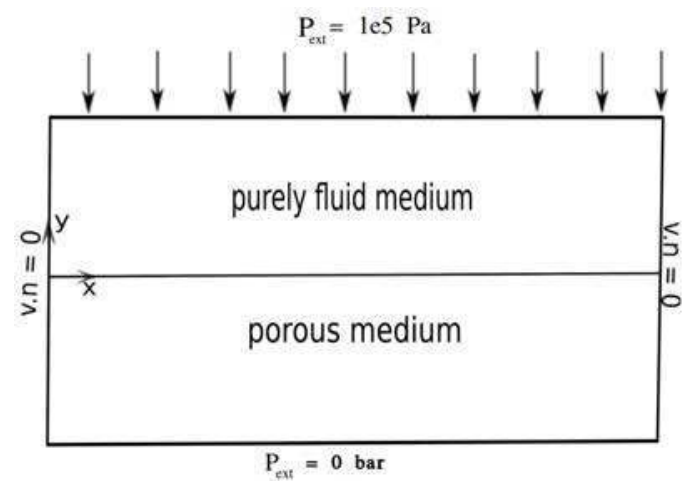

(a)

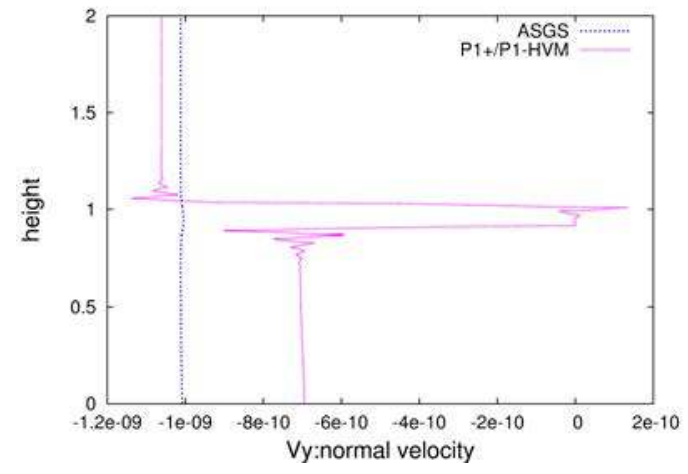

(b)

Fig. 1: Computational domain (a) comparison of normal velocity between P1+/P1-HVM method (b), perpendicular flow $\left(\mathrm{K}=10^{-14} \mathrm{~m}^{2}, \mu=1\right.$ Pa.s, $\left.\alpha=1\right)$

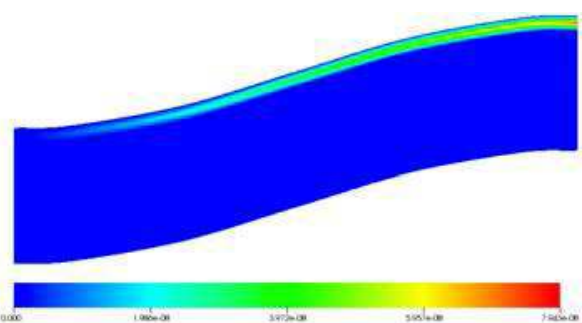

(a)
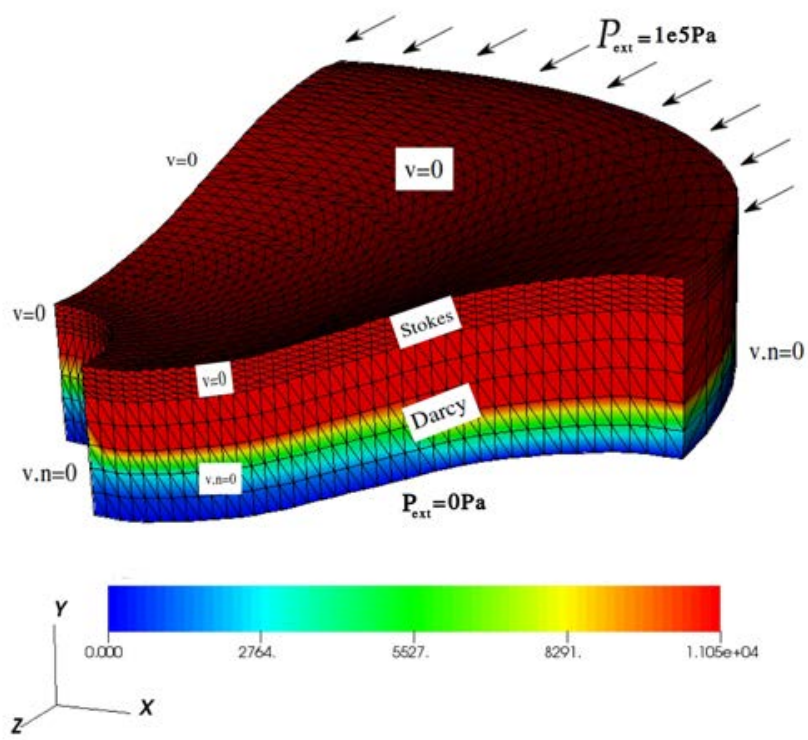

(c)

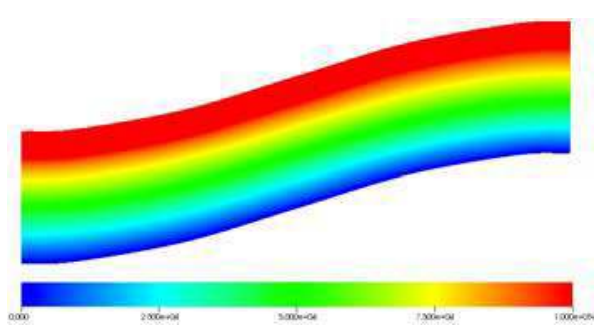

(b)

Fig. 2: Isovalues of velocity (a) and pressure (b) for 2D simulation and 3D simulation (c) for a complex flow with curved interface $\left(\mathrm{K}=10^{-9} \mathrm{~m}^{2}, \mu=1\right.$ Pa.s, $\left.\alpha=1\right)$ 

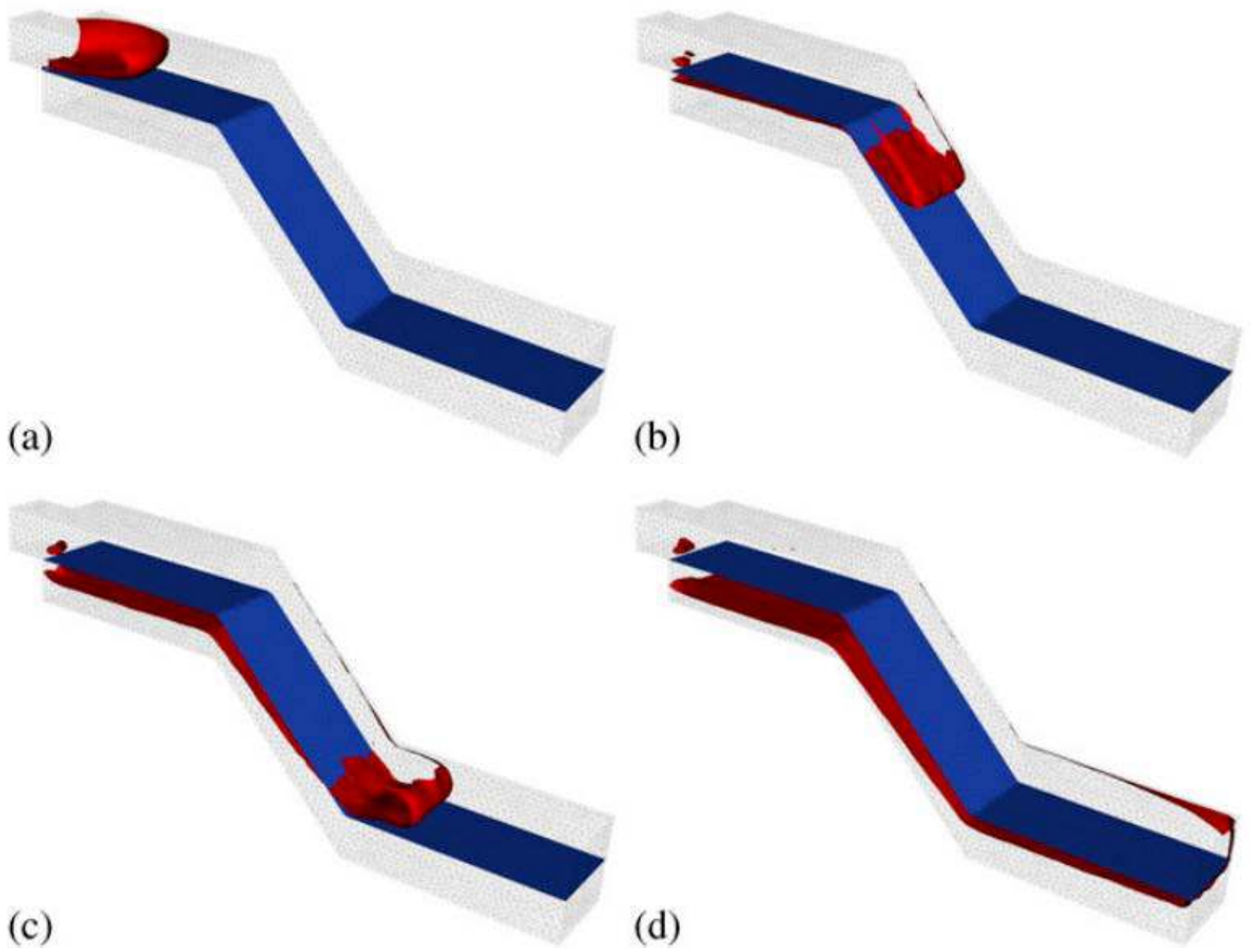

Fig. 3: 3D simulation of the process by resin infusion: flow front advancement during the resin injection.

\section{Conclusions}

The monolithic approach has been developped to solve Stokes-Darcy coupled problem. A stabilized finite element method has been proposed to stabilize Stokes, Darcy and Stokes/Darcy coupled problem in the case where the Brezzi-Babuska stability condition is not fulfilled. This stabilization method is based on a variational multiscale technique called ASGS method. Convergence of this method was validated and compared with another approach [2] showing the accuracy of the results. Also, it was shown that the method could be used for more complex geometries and with a wide range of permeability, which is essential in the industrial applications. The current work focuses more specifically on the simulation of the resin infusion process: coupling the moving flow front and the deformation of preforms, and coupling the mechanical problem with the thermo-physico-chemistry of the resin. 


\section{References}

[1] P. Celle, S. Drapier, and J-M. Bergheau: European Journal of Mechanics, Vol. 27 (2008), p. 647661.

[2] G. Pacquaut, J. Bruchon, N. Moulin, and S. Drapier: International Journal for Numerical Methods in Fluids, Vol. 69 (2012), p. 459-480.

[3] S.Badia and R.Codina: Computer Methods in Applied Mechanics and Engineering, Vol. 199 (2010), p. 654-667.

[4] S.Badia and R.Codina: SIAM journal on Numerical Analysis, Vol. 47 (2008), p. 1971-2000. 